The University of Southern Mississippi

The Aquila Digital Community

Faculty Publications

6-1-2021

\title{
Broadband Infrared Photodetection Using a Narrow Bandgap Conjugated Polymer
}

Jarrett $\mathrm{H}$. Vella

Wright-Patterson AFB

Lifeng Huang

University of Southern Mississippi

Naresh Eedugurala

University of Southern Mississippi, naresh.eedugurala@usm.edu

Kevin S. Mayer

University of Southern Mississippi, Kevin.Mayer@usm.edu

Tse Nga Ng

The Electrical and Computer Engineering Department, tnn046@ucsd.edu

See next page for additional authors

Follow this and additional works at: https://aquila.usm.edu/fac_pubs

\section{Recommended Citation}

Vella, J., Huang, L., Eedugurala, N., Mayer, K., Ng, T., Azoulay, J. (2021). Broadband Infrared Photodetection Using a Narrow Bandgap Conjugated Polymer. Science Advances, 7(24).

Available at: https://aquila.usm.edu/fac_pubs/18834

This Article is brought to you for free and open access by The Aquila Digital Community. It has been accepted for inclusion in Faculty Publications by an authorized administrator of The Aquila Digital Community. For more information, please contact Joshua.Cromwell@usm.edu. 


\section{Authors}

Jarrett H. Vella, Lifeng Huang, Naresh Eedugurala, Kevin S. Mayer, Tse Nga Ng, and Jason D. Azoulay 


\section{Broadband infrared photodetection using a narrow bandgap conjugated polymer}

\author{
Jarrett H. Vella ${ }^{1 \dagger}$, Lifeng Huang ${ }^{2 \dagger}$, Naresh Eedugurala ${ }^{2}$, Kevin S. Mayer $^{2}$, \\ Tse Nga $\mathrm{Ng}^{3}$, Jason D. Azoulay ${ }^{2}$
}

\begin{abstract}
Photodetection spanning the short-, mid-, and long-wave infrared (SWIR-LWIR) underpins modern science and technology. Devices using state-of-the-art narrow bandgap semiconductors require complex manufacturing, high costs, and cooling requirements that remain prohibitive for many applications. We report high-performance infrared photodetection from a donor-acceptor conjugated polymer with broadband SWIR-LWIR operation. Electronic correlations within the $\pi$-conjugated backbone promote a high-spin ground state, narrow bandgap, long-wavelength absorption, and intrinsic electrical conductivity. These previously unobserved attributes enabled the fabrication of a thin-film photoconductive detector from solution, which demonstrates specific detectivities greater than $2.10 \times 10^{9}$ Jones. These room temperature detectivities closely approach those of cooled epitaxial devices. This work provides a fundamentally new platform for broadly applicable, low-cost, ambient temperature infrared optoelectronics.
\end{abstract}

\section{INTRODUCTION}

The detection of infrared (IR) light forms the foundation for modern industrial, scientific, energy, medical, and defense applications. Inorganic semiconductors spanning different wavelength $(\lambda)$ regimes and complex manufacturing systems are the basis for technologies operating beyond the cutoff of silicon $\left(\lambda_{c} \sim 1.1 \mu \mathrm{m}\right)$. State-of-the-art IR photodetectors are often diodes based off of III-V semiconductors; for example, indium gallium arsenide (InGaAs) covers up to $\lambda=2.4 \mu \mathrm{m}$ in the shortwave IR (SWIR; $\lambda=1$ to $3 \mu \mathrm{m}$ ); indium antimonide (InSb), the midwave IR (MWIR; $\lambda=3$ to $5 \mu \mathrm{m}$ ); and mercury cadmium telluride (HgCdTe), the longwave IR (LWIR; $\lambda=8$ to $14 \mu \mathrm{m}$ ) (1). These detectors are fabricated using high-temperature epitaxial growth and suffer from limited modularity and intrinsic fragility, generally require cryogenic cooling, and are incompatible with complementary metal-oxide semiconductor (CMOS) technologies (2-4). Similar constraints are associated with other mature technologies such as doped heterostructures of III-V semiconductors, quantum wells, and type-II superlattices $(5,6)$. There remains a need for new semiconductors with figures of merit similar to III-V materials, but that also provide substantial cost reductions, scalable fabrication, heterogeneous integration, geometric forms, and mechanical properties that are not available using current technologies.

Consequently, alternative materials have long been researched, such as various allotropes of carbon (7), graphene (8), other twodimensional (2D) materials $(9,10)$, transition metal dichalcogenides, black arsenic phosphorus (b-AsP) (11), and solution-processable conjugated polymers (CPs), colloidal quantum dots (CQDs), and metalhalide perovskites (MHPs) (12-18). In general, the long-wavelength operation of solution-processable materials such as CPs and MHPs is limited to the near IR (NIR), while other colloidal materials such as CQDs progress further into the IR (19). These materials have enabled tremendous advancements that overcome classical photodetection

\footnotetext{
${ }^{1}$ Sensors Directorate, Air Force Research Laboratory, Wright-Patterson Air Force Base, Dayton, OH 45433, USA. ${ }^{2}$ Center for Optoelectronic Materials and Devices, School of Polymer Science and Engineering, The University of Southern Mississippi, Hattiesburg, MS 39406, USA. ${ }^{3}$ Department of Electrical and Computer Engineering, University of California San Diego, 9500 Gilman Drive, La Jolla, CA 92093-0407, USA. *Corresponding author. Email: jason.azoulay@usm.edu

tThese authors contributed equally to this work.
}

limitations. For example, solution-processed PbS CQDs operating at $1.3 \mu \mathrm{m}$ show higher specific detectivities $\left(D^{*}\right)$ at room temperature than cooled epitaxial devices that results from a large photoconductive gain (20), while ambient temperature MWIR-LWIR operation has been demonstrated using b-AsP $(\lambda=3$ to $8.05 \mu \mathrm{m})(11)$ and $\mathrm{PdSe}_{2}$ (up to $10.6 \mu \mathrm{m})(21)$. However, several challenges remain to facilitate the technical maturation and practical application of alternative narrow bandgap materials such as high dark current and noise levels, low light absorption, chemical instability, and complex manufacturing approaches. Thus, cooled epitaxial semiconductors remain the dominant technology throughout the SWIR-LWIR spectral regions.

The realization of narrow bandgap semiconductors with strong IR absorption, appropriate electronic properties, solution-processability, and stability continues to remain a considerable challenge, and organic materials that offer alternatives to inorganics and graphene have yet to be found. While CPs offer the most versatility in terms of their ease of processing, scalability, cost, and structural tunability, the spectral ranges in which they operate remain limited. State-ofthe-art polymer photodetectors composed of photodiodes and phototransistors largely operate in the visible to $\operatorname{NIR}(\lambda=0.4$ to $1 \mu \mathrm{m})$ $(12-16,22,23)$. There are a few studies partially extending CPs into the SWIR that combine NIR bandgaps with low-energy absorption tails, or studies that alter the density of states in donor-acceptor (DA) blends (24-27). A smaller number of studies describe organicbased MWIR photodetectors such as a CQD-polymer hybrid (28) and composite of poly(3,4-ethylenedioxythiophene)-poly(4styrenesulfonate) and graphene (29); however, the CQD and graphene are responsible for the longer wavelength photoresponse. Organic materials have also been applied in thermal detectors such as graphenebased bolometers (30) and polymer-based pyroelectric detectors, albeit with much lower response speeds (31).

Despite a vast body of knowledge and practice, controlling the bandgaps of CPs at very low energies remains a considerable challenge owing to an absence of design rules that connect chemical, electronic, and structural heterogeneities with the degree of electronic correlation and energetic disorder in these materials. Moreover, current organic photodiode strategies are unlikely to result in a useful IR response by virtue of their high nonradiative recombination rates $(23,32)$. While most CPs exhibit closed-shell structures that 
accommodate their $\pi$-electrons in bonding orbitals, recent reports have demonstrated that DA copolymers with extensive $\pi$-delocalization, quinoidal character, and very narrow bandgaps show open-shell electronic structures. The rich behavior of these materials emanates from the collective properties of $\pi$-electrons along the conjugated backbone and diminished intramolecular electron pairing, which enables new applications (33-36). The recent development of highspin DA CPs has produced polymers with electronic bandgaps in the SWIR-MWIR with absorption extending well into the LWIR $(34,35)$. Here, we demonstrate the first example of a photoconductive detector that uses an open-shell DA CP active layer that senses SWIR, MWIR, and LWIR photons.

\section{RESULTS}

Figure 1A displays the copolymer used in this study. We previously demonstrated that DA CPs based on alternating cyclopentadithiophenethiadiazoloquinoxaline (TQ) frameworks give rise to high-spin ground states, very narrow optical bandgaps $\left(0.2 \mathrm{eV}<E_{\mathrm{g}}{ }^{\text {ppt }}<1 \mathrm{eV}\right)$, intrinsic electrical conductivities $(\sigma)$ that range from $\sim 10^{-6}$ to $8 \mathrm{~S} \mathrm{~cm}^{-1}$, and robust stability emanating from strong electronic correlations and extensive $\pi$-delocalization $(34,35)$. This motivated our development of poly(4-(4,4-dimethyl-4H-cyclopenta[2,1-b:3,4- $\left.b^{\prime}\right]$ dithiophen-2-yl)6,7-bis(5-hexadecylthiophen-2-yl)-[1,2,5] thiadiazolo[3,4-g]quinoxaline), a neutral high-spin DA CP in which macromolecular structure and electronic topology coalesce to afford a very narrow $E_{\mathrm{g}}{ }^{\text {opt }}$ of $<0.10 \mathrm{eV}$. The polymer was synthesized using a microwave-assisted

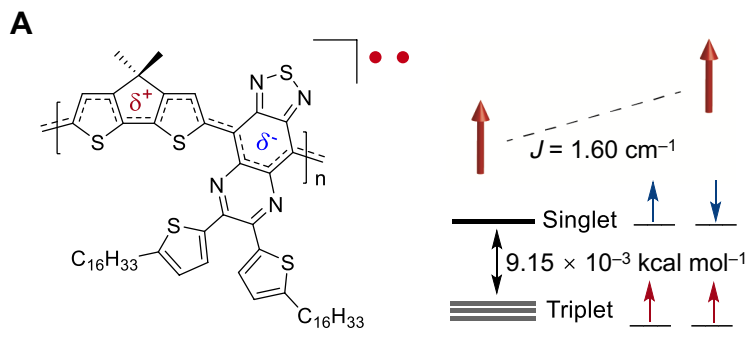

B

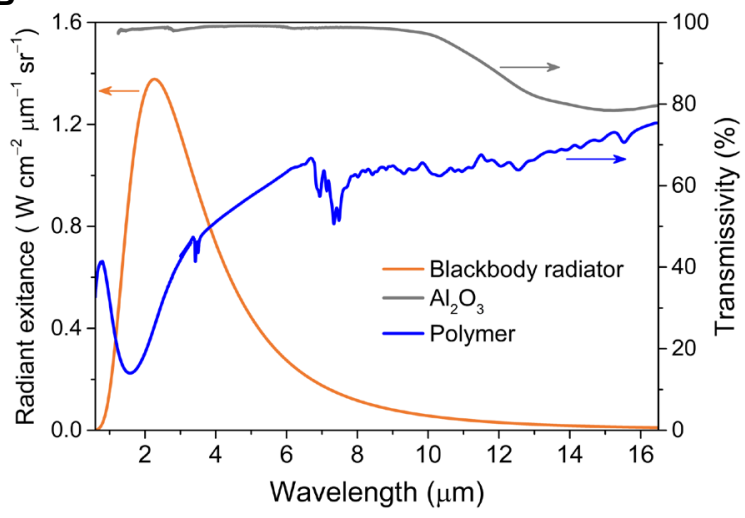

Fig. 1. Infrared absorption of the high-spin conjugated polymer. (A) Molecular and electronic structure of the narrow bandgap polymer. The measured magnetic properties exhibit a high-to-low spin energy gap of $9.15 \times 10^{-3} \mathrm{kcal} \mathrm{mol}^{-1}$ and exchange coupling constant $(J)$ of $1.60 \mathrm{~cm}^{-1}$ that is consistent with ferromagnetic coupling between spins. (B) Transmission spectra of a thin film spin-coated from chlorobenzene onto an $\mathrm{NaCl}$ substrate (blue trace), calculated exitance of a $1000^{\circ} \mathrm{C}$ blackbody radiator (orange), and spectral transmissivity of the $\mathrm{Al}_{2} \mathrm{O}_{3}$ encapsulant (gray).
Stille cross-coupling copolymerization (see the Supplementary Materials). A salient design feature is a TQ acceptor in which ancillary thiophene substituents enable a further modification of the frontier orbital energetics and strategic positioning of solubilizing $-\mathrm{C}_{16} \mathrm{H}_{33}$ side chains. This serves to minimize backbone torsion and promote a high degree of electronic coherence along the $\pi$-conjugated backbone (fig. S5). Variable-temperature electron paramagnetic resonance spectroscopy demonstrates that the polymer exhibits a singlet-triplet energy splitting $\left(\Delta E_{\mathrm{ST}}\right)$ of $9.15 \times 10^{-3} \mathrm{kcal} \mathrm{mol}^{-1}$ and exchange coupling coefficient $(J)$ of $1.60 \mathrm{~cm}^{-1}$ (fig. S6). Thus, the polymer has ferromagnetically coupled spins $(J>0$, parallel $)$ and a $\Delta E_{\mathrm{ST}}$ that allows for thermal population of a nearly degenerate low-spin state with antiferromagnetic interactions between $\pi$-electrons (Fig. 1A). The value of $\Delta E_{\mathrm{ST}}$ means that approximately $75 \%$ of spins (Boltzmann population ratio of 0.98 ) occupy the lower-energy, triply degenerate (high-spin) manifold at room temperature.

The extensive $\pi$-conjugation is evident from a bandgap that is among the lowest obtained for conjugated organic materials and the lowest for a solution-processable material. The polymer thin film demonstrates an absorption maximum $\left(\lambda_{\max }\right)$ of $1.67 \mu \mathrm{m}$ and an electronic absorption tail extending throughout the MWIR-LWIR, in which vibrational features are superimposed (Fig. 1B). Fourier transform IR (FTIR) spectroscopy is consistent with an $E_{\mathrm{g}}{ }^{\text {opt }}<0.10 \mathrm{eV}$ as estimated from the absorption onset of the thin film (fig. S5). The offset from unity transmission of 46 to $63 \%$ in the MWIR and 68 to $76 \%$ in the LWIR is far stronger than monolayer graphene in these spectral regions (8). Charge transport measurements show linear currentvoltage $(I-V)$ characteristics, consistent with $\sigma=9.72 \times 10^{-5} \mathrm{~S} \mathrm{~cm}^{-1}$ in the absence of dopants (fig. S7). The ohmic transport can be associated with the low energetic barrier for thermal excitation of free carriers, as in other photoresponsive narrow bandgap semiconductors, along with extensive spin and $\pi$-delocalization (34).

These properties were leveraged to realize a photoconductive detector, wherein absorption in the active layer generates additional charge carriers, which, under the action of an external DC bias, drift to the appropriate electrodes and generate a measurable change in current. The detector active area is schematically depicted in Fig. 2A as a capacitor in parallel with a variable resistor. A load resistor is in series with the detector, which represents the resistance of the external measurement circuitry. Figure $2 \mathrm{~B}$ depicts a cross section of the proof-of-concept detector. The device consists of prepatterned gold electrodes separated by a $60 \mu \mathrm{m} \times 1 \mathrm{~mm}$ spacing that acts as the detector active area with a transmission line leading away from each electrode. The polymer was spin-coated onto the detector structure, followed by active area encapsulation with a $100 \mathrm{~nm}$ amorphous alumina film that is largely transparent throughout the SWIR and MWIR, with only slight LWIR attenuation (Fig. 1B). The detector was then mounted in a ceramic leadless chip carrier (LCC; Fig. 2C), with gold wire bonds connecting the LCC to the chip.

We initially examined devices with films of varying thicknesses of 76 and $1078 \mathrm{~nm}$. Conductivity measurements of the thinner film showed $\sigma=9.72 \times 10^{-5} \mathrm{~S} \mathrm{~cm}^{-1}$, while $\sigma$ increased to $5.68 \times 10^{-3} \mathrm{~S} \mathrm{~cm}^{-1}$ in the thicker film (fig. S7), consistent with differences in processing conditions (see the Supplementary Materials). Under an applied DC bias of $+5 \mathrm{~V}$, the dark current of the fully packaged detector changed from 30 to $226 \mathrm{nA}$ as the thickness increased, and the DC resistance changed from 35.7 to 1.26 megaohm. The literature is replete with examples of processing resulting in improved chain organization, thereby enhancing the transport properties of closely 


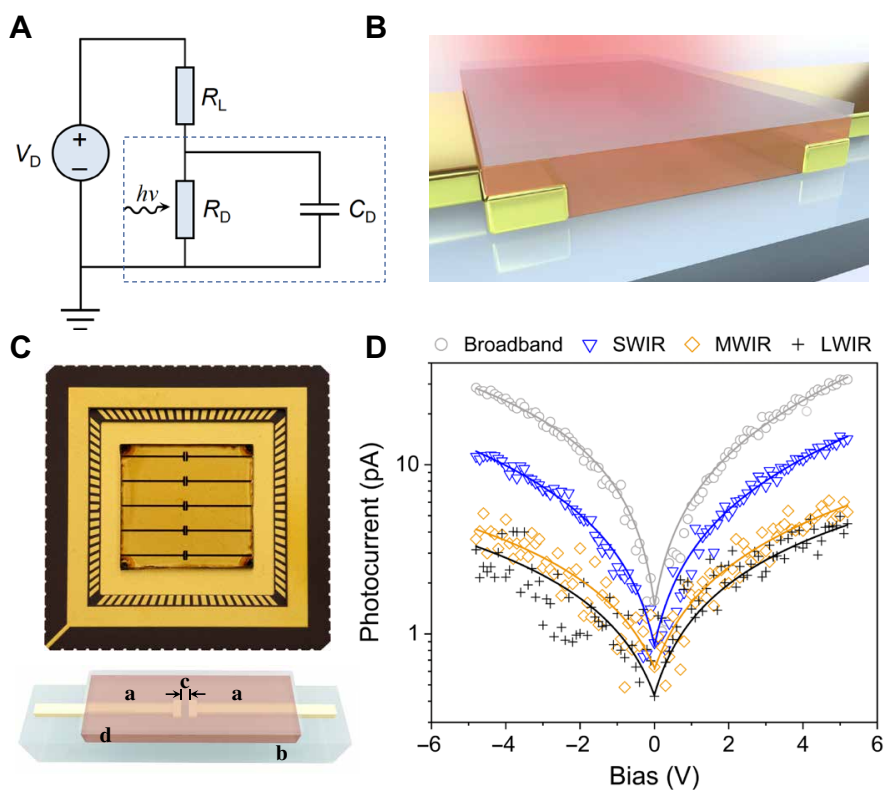

Fig. 2. Device structure and IR photocurrent response. (A) Equivalent circuit of a photoconductive detector: $V_{\mathrm{D}}$ is the DC bias, $R_{\mathrm{L}}$ is the load resistance, $R_{\mathrm{D}}$ is the resistance of the detector element modulated by photon absorption, and $C_{D}$ is the detector capacitance. (B) Schematic illustration of the $60 \mu \mathrm{m} \times 1 \mathrm{~mm}$ detector active area. (C) Single-element photoconductive devices mounted in a ceramic LCC. Inset shows (a) transmission lines, (b) a dielectric substrate, (c) the detector active area, and (d) the boundary of the polymer and dielectric encapsulant. (D) Photocurrent generated under irradiation with a $1000^{\circ} \mathrm{C}$ blackbody without a spectral bandpass filter, using a SWIR bandpass filter $(\lambda=1$ to $3 \mu \mathrm{m})$, a MWIR filter ( $\lambda=3$ to $5 \mu \mathrm{m})$, and a partial LWIR filter $(\lambda=8$ to $12 \mu \mathrm{m})$. The applied bias was $+5 \mathrm{~V}$, and the integrated SWIR, MWIR, and LWIR spectral power is $2.17,1.55$, and $0.255 \mathrm{nW}$, respectively. Photo credit: Lifeng Huang, The University of Southern Mississippi.

related CPs and open-shell conductors (34, 37-39). Aggregation in the thicker film increases long-range polymer ordering resulting in a more highly conductive film (34). The decreased resistance allows more charge carriers generated by Johnson, generationrecombination (G-R), and $1 / f$ mechanisms to be collected (2). While high-dark current noise is a major challenge for modern narrow bandgap semiconductors, these results point toward the potential for substantial optimization because electronic properties and noise characteristics can be modified using various solution-processing techniques and diverse fabrication approaches. For example, possible optimization routes include identifying a more ohmic contact to reduce $1 / f$ noise and establishing improved processing approaches to minimize defects and charge traps to reduce G-R noise (14-17).

Photocurrents of thin-film $(76 \mathrm{~nm})$ detectors were measured by illuminating the detector with a $1000^{\circ} \mathrm{C}$, chopped cavity blackbody radiator (2). The blackbody temperature and emission spectrum were chosen to overlap as much as possible with the polymer absorption (Fig. 1B). The calculated radiant exitance features a maximum intensity at $\lambda=2.29 \mu \mathrm{m}$, compared with an absorption maximum centered at $\lambda=1.67 \mu \mathrm{m}$ for the polymer. Measurements were made without an optical bandpass filter in front of the blackbody (broadband) along with SWIR $(\lambda=1$ to $3 \mu \mathrm{m}), \operatorname{MWIR}(\lambda=3$ to $5 \mu \mathrm{m})$, and LWIR filters $(\lambda=8$ to $12 \mu \mathrm{m})$. Photocurrent-voltage curves for all four spectral regions were linear and relatively symmetric about $0 \mathrm{~V}$, indicating that the material is acting as a resistor, consistent with a photoconductive detection mechanism. Photocurrents of 31.97,
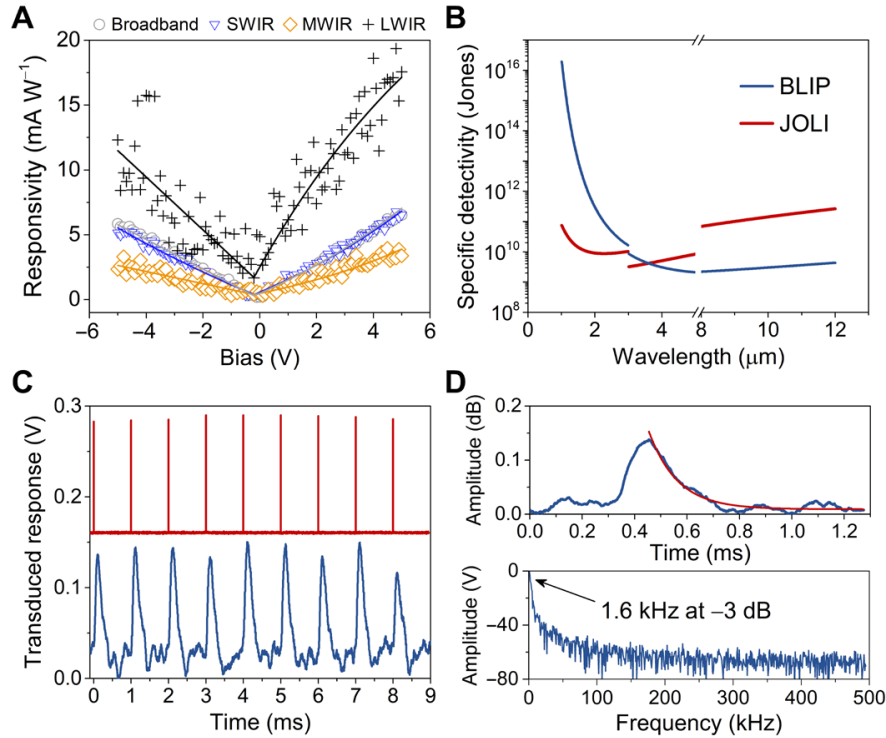

Fig. 3. Performance characteristics of the photoconductive detector at room temperature. (A) Responsivity as a function of applied bias over four IR spectral regions. (B) JOLI $D^{*}$ and BLIP D*. (C) Photoconductive polymer response (blue) and a commercial InGaAs photodiode response substituted for the polymer (red) toward a train of femtosecond laser pulses (1550 nm, 1 kHz, $150 \mathrm{fs}$ ). (D) (Top) Expansion of a single femtosecond detector transient and a single exponential fit (red) gives a 10 to $90 \%$ rise time of $115 \mu \mathrm{s}$ and a decay constant of $109 \mu \mathrm{s}$. (Bottom) Bode plot of the photoconductive detector showing a $-3 \mathrm{~dB}$ bandwidth of $1.6 \mathrm{kHz}$.

$14.21,5.24$, and $4.48 \mathrm{pA}$ were obtained at $+5 \mathrm{~V}$ for broadband, SWIR, MWIR, and LWIR illumination, respectively (Fig. 2D).

The photocurrent data can be converted to responsivity (Fig. 3A) at a fixed bias level by dividing it by the blackbody power in each spectral region. The actual power irradiating the detector is obtained through numerical integration of the calculated blackbody spectrum and is ultimately a function of detector area and distance from the source (solid angle), spectral bandpass filter used, and a form factor that relates the size of the blackbody aperture with the size of the openings in the chopper wheel (2) (see the Supplementary Materials). The broadband power incident on the detector is $4.89 \mathrm{nW}$, and the SWIR, MWIR, and LWIR power is $2.17,1.55$, and $0.255 \mathrm{nW}$, respectively. At $+5 \mathrm{~V}$, responsivities of $6.47,6.33,3.88$, and $15.3 \mathrm{~mA} \mathrm{~W}^{-1}$ were calculated for broadband, SWIR, MWIR, and LWIR regions, respectively. Noise equivalent power (NEP) describes the optical power incident on the detector to give a signal-to-noise ratio of 1 with a $1-\mathrm{Hz}$ bandwidth. The NEP is calculated by dividing the dark current by responsivity. For broadband, SWIR, MWIR, and LWIR regions, $\mathrm{NEP}=72.4,68.1,104$, and $24.3 \mathrm{nW} \mathrm{Hz}^{-1 / 2}$, respectively. Repeated measurements of the device showed no discernible changes in photocurrent over the course of 1 year (fig. S8).

An important figure of merit is the specific detectivity, $D^{*}$, which allows a comparison of performance between different detector technologies. Photoconductive detectors can be limited either by Johnson noise, the Johnson noise limited (JOLI) case (Eq. 1), or background irradiation, the background-limited infrared photodetection (BLIP) condition (Eq. 2) (2). Johnson noise is a noise current induced by the thermal motion of charge carriers in resistive materials. It becomes the limiting noise process when all other noise mechanisms in a photoconductive detector have been minimized and can 
be reduced by cooling. JOLI $D^{*}$ for a photoconductive detector is defined as

$$
D_{\mathrm{PC}, \text { JOLI }}^{*}=\frac{\eta \lambda q_{\mathrm{e}}}{2 h c} G \sqrt{\frac{R_{\mathrm{eq}} A_{\mathrm{d}}}{k T_{\mathrm{d}}}}
$$

where $\eta$ is the detector external quantum efficiency, $\lambda$ is the wavelength, $q_{\mathrm{e}}$ is the elementary charge, $h$ is the Planck's constant, $c$ is the speed of light, $G$ is the detector gain, $R_{\text {eq }}$ is the detector equivalent resistance, $A_{\mathrm{d}}$ is the detector area, $k$ is the Boltzmann constant, and $T_{\mathrm{d}}$ is the detector temperature.

The BLIP condition occurs when the limiting noise is determined by fluctuations in the generation and recombination of charge carriers. The background-limited $D^{*}$ is strongly affected by the incident background irradiance $\left(E_{\mathrm{bkg}}\right)$

$$
D_{\mathrm{PC}, \mathrm{BLIP}}^{*}=\frac{\lambda}{2 h c} \sqrt{\frac{\eta}{E_{\mathrm{bkg}}}}
$$

For an extremely broadband photodetector such as the one described here, the limiting noise mechanism, and thus $D^{*}$, can conceivably shift depending on the region of operation. When using a blackbody radiator to characterize a detector, spectral $D^{*}$ for both cases is calculated using a blackbody-to-spectral conversion factor $u$, which relates the detector responsivity $(\mathfrak{R})$ at a given cutoff wavelength $\left(\lambda_{c}\right)$, to its response under irradiation by a blackbody of temperature $T$

$$
u=\frac{\Re\left(\lambda_{c}, f\right)}{\mathfrak{R}(T, f)}=\frac{\sigma T^{4}}{\frac{h c}{\lambda_{c}} \int_{0}^{\lambda_{c}} M_{\mathrm{q}}(\lambda, T) d \lambda}
$$

It is essentially the ratio of the total photon exitance of a blackbody at a given temperature to its spectral exitance $M_{\mathrm{q}}(\lambda, T)$ up to $\lambda_{c}$, where $\sigma$ defines the Stefan-Boltzmann constant in Eq. $3(2,40)$.
JOLI- and BLIP-limited $D^{*}$ were calculated from the responsivity data with $u$ as a spectral conversion factor (Fig. 3B). In the SWIR, detectivity is limited by Johnson noise $\left(D^{*}=7.53 \times 10^{10}\right.$ to $1.03 \times$ $10^{10}$ Jones). The MWIR region featured a crossover point at $\lambda=$ $3.60 \mu \mathrm{m}$, where the limiting noise mechanism changed to BLIP $\left(D^{*}=3.21 \times 10^{9}\right.$ to $2.10 \times 10^{9}$ Jones). LWIR detectivity was BLIP limited as well, with $D^{*}=2.10 \times 10^{9}$ to $4.34 \times 10^{9}$ Jones. This trendJOLI-limited SWIR, BLIP-limited MWIR, and LWIR-is reasonable. The emission spectrum of a $1000{ }^{\circ} \mathrm{C}$ blackbody radiator is primarily located in the SWIR, while a room temperature blackbody radiates principally in the LWIR. The MWIR transitions from a spectrum dominated by a higher temperature blackbody to a room temperature blackbody.

The response speed and $-3 \mathrm{~dB}$ electrical bandwidth are important figures of merit for photoconductors. The $I-V$ phase angle was $0^{\circ}$, preventing resistance-capacitance time constant measurements. Instead, the detector rise time $\left(\tau_{\mathrm{r}}\right)$ was calculated from the detector impulse response using a femtosecond laser operating at $1.55 \mu \mathrm{m}$. The laser pulse width of $150 \mathrm{fs}$ is much faster than any detector can respond, allowing a direct calculation of $\tau_{\mathrm{r}}$. The photocurrent transients in the polymer are coincident with the laser pulses and repeatable over many cycles (Fig. 3C). Expansion of one of these transients (Fig. 3D) enables calculation of the 10 to $90 \% \tau_{\mathrm{r}}$ of $115 \mu \mathrm{s}$. This gives an optically measured bandwidth of $1.5 \mathrm{kHz}$ following $f=\left(2 \pi \tau_{\mathrm{r}}\right)^{-1}$. Fitting the impulse response decay with a single exponential gives a relaxation time of $109 \mu \mathrm{s}$. Applying a fast Fourier transform to the optical impulse response gives the photocurrent frequency spectrum (Fig. 3D). The frequency at which the responsivity decreases by $-3 \mathrm{~dB}$ is $1.6 \mathrm{kHz}$, which agrees closely with the optically measured bandwidth. Because sample heating through electronphonon collisions occurs over picoseconds, the 150 fs pulse width of the laser precludes any heating of the sample. If the detector had a purely thermal response such as the one obtained through a bolometric mechanism, these measurements would not have produced any meaningful impulse response.

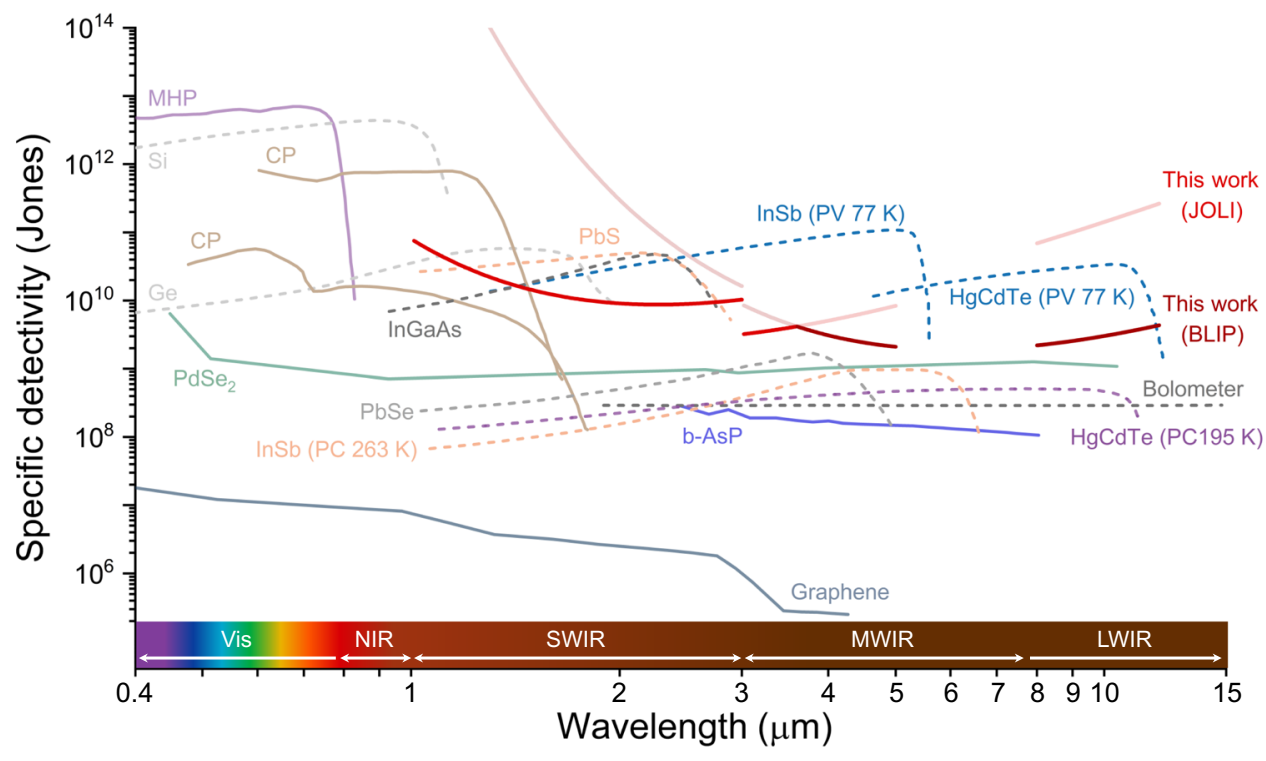

Fig. 4. Spectral $D^{*}$ under JOLI and BLIP conditions for this photoconductive (PC) detector compared with that of other detector technologies. 


\section{DISCUSSION}

At room temperature, and in the absence of optimization, this photoconductive detector is competitive with III-V technologies $(1,2,5)$. For example, uncooled extended InGaAs $\left(\lambda_{c}=2.6 \mu \mathrm{m}\right)$ has peak $D^{*} \sim 10^{10}$ Jones $(1,9)$. Commercial thermoelectrically cooled photoconductive $\operatorname{InSb}(\lambda=\sim 1$ to $6 \mu \mathrm{m})$ and $\operatorname{HgCdTe}(\lambda=\sim 1$ to $12 \mu \mathrm{m})$ detectors show peak $D^{*} \sim 10^{8}$ to $10^{9}$ Jones (1). State-of-the-art photovoltaic $\operatorname{InSb}\left(\lambda_{\mathrm{c}}=5 \mu \mathrm{m}\right)$ and $\mathrm{HgCdTe}\left(\lambda_{\mathrm{c}}=10 \mu \mathrm{m}\right)$ detectors cryogenically cooled to $77 \mathrm{~K}$ show peak $D^{*} \sim 10^{11}$ Jones and $D^{*} \sim 10^{10}$ Jones, respectively (1). However, our detector compares with many commercial systems. When compared to alternative materials for III-V semiconductors, the polymer has a $D^{*}$ competitive with $\mathrm{PbS}(\lambda=\sim 1$ to $3 \mu \mathrm{m}$, peak $D^{*} \sim 10^{10}$ Jones) and is more sensitive than $\operatorname{PbSe}(\lambda=\sim 1.5$ to $4.8 \mu \mathrm{m}$, peak $D^{*} \sim 10^{9}$ Jones) in the MWIR (Fig. 4) (1). In the LWIR, the polymer performs far better than graphene and microbolometers and outperforms emerging materials such as b-AsP $\left(D^{*} \sim 10^{8}\right.$ Jones at $\lambda=8.05 \mu \mathrm{m})(11)$ and $\mathrm{PdSe}_{2}\left(D^{*} \sim 1 \times 10^{9}\right.$ Jones at $\lambda=10.6 \mu \mathrm{m})(21)$.

This work demonstrates the first example of broadband IR photodetection using a DA CP in spectral regions, which organic materials outside of graphene have failed to access. This material platform provides robust stability, control of the bandgap at very low energies, facile synthetic tuning of optoelectronic properties, solution processability, and room temperature operation-properties that are not synergistically achievable from other semiconductors. These attributes, along with the inherent simplicity of detector fabrication, provide an alternative means to transduce IR photons and enable fundamentally new device technologies.

\section{MATERIALS AND METHODS \\ Photoconductor fabrication}

Synthetic quartz-coated glass substrates were sequentially cleaned using 2\% Hellmanex detergent in deionized (DI) water, DI water, acetone, and isopropyl alcohol for $10 \mathrm{~min}$ using sonication, followed by drying in an oven. The substrates were then treated in a $\mathrm{UV}$-ozone cleaner for $30 \mathrm{~min}$. Gold electrodes $(60 \mathrm{~nm})$ were thermally evaporated at $1 \times 10^{-7}$ torr using a shadow mask. The electrodes were separated by a $60 \mu \mathrm{m} \times 1 \mathrm{~mm}$ spacing that acts as the detector active area with a transmission line leading away from each electrode as shown in Fig. 2C. The polymer was dissolved in chloroform at $58^{\circ} \mathrm{C}$ to make a solution of $10 \mathrm{mg} \mathrm{ml}^{-1}$ in a nitrogen atmosphere. The polymer solution was spin-coated onto the substrate at $1000 \mathrm{rpm}$ for $60 \mathrm{~s}$, resulting in a $76 \mathrm{~nm}$ thick film. Next, another shadow mask was placed over the center of the substrate, followed by deposition of $100 \mathrm{~nm}$ alumina at $1 \times 10^{-7}$ torr over the center of the detector (layer $\mathrm{d}$ in Fig. 2C) using electron beam evaporation.

\section{Device characterization}

The detector chip was mounted in a ceramic LCC. Ultrasonically extruded, gold wires connected the gold contact pads on the chip to the LCC leads. The LCC was then mounted in a custom-designed, printed circuit board (PCB). For responsivity measurements, the detector was illuminated with a cavity blackbody radiator (Santa Barbara Infrared Inc.) at $1000^{\circ} \mathrm{C}$ equipped with a mechanical chopper. The PCB was connected by way of triaxial cables to a low-noise current preamplifier (SR570, Stanford Research Systems) and lock-in amplifier (SR830, Stanford Research Systems). Data acquisition was computer-controlled using a custom written program. A Spectra-Physics
Solstice femtosecond laser $(1-\mathrm{kHz}$ repetition rate, $90 \mathrm{fs}$ pulse width, $800 \mathrm{~nm}$ ) was used to pump a TOPAS optical parametric amplifier to produce a wavelength of $1550 \mathrm{~nm}$ with a pulse width of $150 \mathrm{fs}$ and a repetition rate of $1 \mathrm{kHz}$. A pair of linear polarizers was used to control the laser power. The photocurrent was routed by way of triaxial cables to an SR570 current preamplifier and voltage amplifier (SR560, Stanford Research Systems) and then finally to a $300 \mathrm{MHz}$ digitizing oscilloscope (Tektronix DPO3034). Electrical phase angle measurements were made using an Agilent E4980 LCR meter.

\section{SUPPLEMENTARY MATERIALS}

Supplementary material for this article is available at http://advances.sciencemag.org/cgi/ content/full/7/24/eabg2418/DC1

\section{REFERENCES AND NOTES}

1. A. Rogalski, Infrared and Terahertz Detectors (CRC Press, 2019).

2. E. L. Dereniak, G. D. Boreman, Infrared Detectors and Systems (Wiley, 1996).

3. W. Lei, J. Antoszewski, L. Faraone, Progress, challenges, and opportunities for $\mathrm{HgCdTe}$ infrared materials and detectors. Appl. Phys. Rev. 2, 041303 (2015).

4. A. Rogalski, J. Antoszewski, L. Faraone, Third-generation infrared photodetector arrays. J. Appl. Phys. 105, 091101 (2009).

5. P. Martyniuk, J. Antoszewski, M. Martyniuk, L. Faraone, A. Rogalski, New concepts in infrared photodetector designs. Appl. Phys. Rev. 1, 041102 (2014).

6. A. Rogalski, P. Martyniuk, M. Kopytko, InAs/GaSb type-ll superlattice infrared detectors: Future prospect. Appl. Phys. Rev. 4, 031304 (2017).

7. M. Richter, T. Heumüller, G. J. Matt, W. Heiss, C. J. Brabec, Carbon photodetectors: The versatility of carbon allotropes. Adv. Energy Mater. 7, 1601574 (2017).

8. B. Y. Zhang, T. Liu, B. Meng, X. Li, G. Liang, X. Hu, Q. J. Wang, Broadband high photoresponse from pure monolayer graphene photodetector. Nat. Commun. 4, 1811 (2013).

9. A. Rogalski, M. Kopytko, P. Martyniuk, Two-dimensional infrared and terahertz detectors: Outlook and status. Appl. Phys. Rev. 6, 021316 (2019).

10. F. Wang, Z. Wang, L. Yin, R. Cheng, J. Wang, Y. Wen, T. A. Shifa, F. Wang, Y. Zhang, X. Zhan, $J$. He, 2D library beyond graphene and transition metal dichalcogenides: A focus on photodetection. Chem. Soc. Rev. 47, 6296-6341 (2018).

11. M. Long, A. Gao, P. Wang, H. Xia, C. Ott, C. Pan, Y. Fu, E. Liu, X. Chen, W. Lu, T. Nilges, J. Xu, $X$. Wang, W. Hu, F. Miao, Room temperature high-detectivity mid-infrared photodetectors based on black arsenic phosphorus. Sci. Adv. 3, e1700589 (2017).

12. G. Simone, M. J. Dyson, S. C. J. Meskers, R. A. J. Janssen, G. H. Gelinck, Organic photodetectors and their application in large area and flexible image sensors: The role of dark current. Adv. Funct. Mater. 30, 1904205 (2020).

13. F. P. García de Arquer, A. Armin, P. Meredith, E. H. Sargent, Solution-processed semiconductors for next-generation photodetectors. Nat. Rev. Mater. 2, 16100 (2017).

14. K.-J. Baeg, M. Binda, D. Natali, M. Caironi, Y.-Y. Noh, Organic light detectors: Photodiodes and phototransistors. Adv. Mater. 25, 4267-4295 (2013)

15. Z. Wu, Y. Zhai, H. Kim, J. D. Azoulay, T. N. Ng, Emerging design and characterization guidelines for polymer-based infrared photodetectors. Acc. Chem. Res. 51, 3144-3153 (2018).

16. C. Wang, X. Zhang, W. Hu, Organic photodiodes and phototransistors toward infrared detection: Materials, devices, and applications. Chem. Soc. Rev. 49, 653-670 (2020).

17. Y. Xu, Q. Lin, Photodetectors based on solution-processable semiconductors: Recent advances and perspectives. Appl. Phys. Rev. 7, 011315 (2020).

18. L. Dou, Y. Yang, J. You, Z. Hong, W.-H. Chang, G. Li, Y. Yang, Solution-processed hybrid perovskite photodetectors with high detectivity. Nat. Commun. 5, 5404 (2014).

19. S. B. Hafiz, M. Scimeca, A. Sahu, D.-K. Ko, Colloidal quantum dots for thermal infrared sensing and imaging. Nano Converg. 6, 7 (2019).

20. G. Konstantatos, I. Howard, A. Fischer, S. Hoogland, J. Clifford, E. Klem, L. Levina, E. H. Sargent, Ultrasensitive solution-cast quantum dot photodetectors. Nature 442, 180-183 (2006).

21. M. Long, Y. Wang, P. Wang, X. Zhou, H. Xia, C. Luo, S. Huang, G. Zhang, H. Yan, Z. Fan, X. Wu, X. Chen, W. Lu, W. Hu, Palladium diselenide long-wavelength infrared photodetector with high sensitivity and stability. ACS Nano 13, 2511-2519 (2019).

22. L. Dou, Y. Liu, Z. Hong, G. Li, Y. Yang, Low-bandgap near-IR conjugated polymers/ molecules for organic electronics. Chem. Rev. 115, 12633-12665 (2015).

23. J.-L. Hou, A. Fischer, S.-C. Yang, J. Benduhn, J. Widmer, D. Kasemann, K. Vandewal, K. Leo, Plasmon-induced sub-bandgap photodetection with organic Schottky diodes. Adv. Funct. Mater. 26, 5741-5747 (2016)

24. B. Siegmund, A. Mischok, J. Benduhn, O. Zeika, S. Ullbrich, F. Nehm, M. Böhm, D. Spoltore H. Fröb, C. Körner, K. Leo, K. Vandewal, Organic narrowband near-infrared 
photodetectors based on intermolecular charge-transfer absorption. Nat. Commun. 8 , 15421 (2017).

25. W. Yao, Z. Wu, E. Huang, L. Huang, A. E. London, Z. Liu, J. D. Azoulay, T. N. Ng, Organic bulk heterojunction infrared photodiodes for imaging out to $1300 \mathrm{~nm}$. ACS Appl. Electron. Mater. 1, 660-666 (2019).

26. X. Gong, M. Tong, Y. Xia, W. Cai, J. S. Moon, Y. Cao, G. Yu, C.-L. Shieh, B. Nilsson, A. J. Heeger, High-detectivity polymer photodetectors with spectral response from $300 \mathrm{~nm}$ to $1450 \mathrm{~nm}$. Science 325, 1665-1667 (2009).

27. A. E. London, L. Huang, B. A. Zhang, M. B. Oviedo, J. Tropp, W. Yao, Z. Wu, B. M. Wong, T. N. Ng, J. D. Azoulay, Donor-acceptor polymers with tunable infrared photoresponse. Polym. Chem. 8, 2922-2930 (2017).

28. A. D. Stiff-Roberts, K. R. Lantz, R. Pate, Room-temperature, mid-infrared photodetection in colloidal quantum dot/conjugated polymer hybrid nanocomposites: A new approach to quantum dot infrared photodetectors. J. Phys. D Appl. Phys. 42, 234004 (2009).

29. M. Zhang, J. T. W. Yeow, A flexible, scalable, and self-powered mid-infrared detector based on transparent PEDOT: PSS/graphene composite. Carbon 156, 339-345 (2020).

30. A. Blaikie, D. Miller, B. J. Alemán, A fast and sensitive room-temperature graphene nanomechanical bolometer. Nat. Commun. 10, 4726 (2019).

31. S. Ebrahim, A. M. Elshaer, M. Soliman, M. B. Tayl, Pyroelectric infrared detector based on polyaniline/polyvinylidene fluoride blend. Sens. Actuat. A Phys. 238, 389-396 (2016).

32. D. B. Sulas, A. E. London, L. Huang, L. Xu, Z. Wu, T. N. Ng, B. M. Wong, C. W. Schlenker, J. D. Azoulay, M. Y. Sfeir, Preferential charge generation at aggregate sites in narrow band gap infrared photoresponsive polymer semiconductors. Adv. Opt. Mater. 6, 1701138 (2018).

33. K. Wang, L. Huang, N. Eedugurala, S. Zhang, M. A. Sabuj, N. Rai, X. Gu, J. D. Azoulay, T. N. Ng, Wide potential window supercapacitors using open-shell donor-acceptor conjugated polymers with stable n-doped states. Adv. Energy Mater. 9, 1902806 (2019).

34. L. Huang, N. Eedugurala, A. Benasco, S. Zhang, K. S. Mayer, D. J. Adams, B. Fowler, M. M. Lockart, M. Saghayezhian, H. Tahir, E. R. King, S. Morgan, M. K. Bowman, X. Gu, J. D. Azoulay, Open-shell donor-acceptor conjugated polymers with high electrical conductivity. Adv. Funct. Mater. 30, 1909805 (2020).

35. A. E. London, H. Chen, M. A. Sabuj, J. Tropp, M. Saghayezhian, N. Eedugurala, B. A. Zhang, Y. Liu, X. Gu, B. M. Wong, N. Rai, M. K. Bowman, J. D. Azoulay, A high-spin ground-state donor-acceptor conjugated polymer. Sci. Adv. 5, eaav2336 (2019).

36. Z. X. Chen, Y. Li, F. Huang, Persistent and stable organic radicals: Design, synthesis, and applications. Chem 7, 288-332 (2021).

37. M. Li, C. An, W. Pisula, K. Müllen, Cyclopentadithiophene-benzothiadiazole donoracceptor polymers as prototypical semiconductors for high-performance field-effect transistors. Acc. Chem. Res. 51, 1196-1205 (2018).
38. M. Chang, G. T. Lim, B. Park, E. Reichmanis, Control of molecular ordering, alignment, and charge transport in solution-processed conjugated polymer thin films. Polymers $\mathbf{9}$ 212 (2017).

39. Y. Joo, V. Agarkar, S. H. Sung, B. M. Savoie, B. W. Boudouris, A nonconjugated radical polymer glass with high electrical conductivity. Science 359, 1391-1395 (2018).

40. A. Daniels, Field Guide to Infrared Systems, Detectors, and FPAs (SPIE, 2018).

41. M. Wang, M. J. Ford, C. Zhou, M. Seifrid, T.-Q. Nguyen, G. C. Bazan, Linear conjugated polymer backbones improve alignment in nanogroove-assisted organic field-effect transistors. J. Am. Chem. Soc. 139, 17624-17631 (2017).

42. Y. Tsubata, T. Suzuki, Y. Yamashita, T. Mukai, T. Miyashi, Tetracyanoquinodimethanes fused with 1,2,5-thiadiazole and pyrazine units. Heterocycles 33, 337-348 (1992).

43. T. V. Richter, C. H. Braun, S. Link, M. Scheuble, E. J. W. Crossland, F. Stelzl, U. Würfel, S. Ludwigs, Regioregular polythiophenes with alkylthiophene side chains. Macromolecules 45, 5782-5788 (2012).

44. B. Bleaney, Anomalous paramagnetism of copper acetate. Rev. Mod. Phys. 25, 161-162 (1953).

Acknowledgments: J.H.V. wishes to thank G. Ariyanwansa, C. Reyner, J. Derov, and M. I. Vaki for the many helpful discussions. Funding: The work performed at The University of Southern Mississippi was made possible through the Air Force Office of Scientific Research (AFOSR) under support provided by the Organic Materials Chemistry Program (grant FA9550-20-10353, program manager: K. Caster) and the National Science Foundation (OIA-1757220). Use of the Stanford Synchrotron Radiation Lightsource, SLAC National Accelerator Laboratory, is supported by the U.S. Department of Energy, Office of Science, Office of Basic Energy Sciences under Contract No. DE-AC02-76SF00515. Author contributions: N.E. and K.S.M. synthesized and characterized the polymer. L.H. fabricated the devices, and J.H.V. characterized the infrared photoresponse. L.H., J.H.V., and T.N.N. analyzed and interpreted the data. All authors contributed to the discussion of results and writing of the manuscript. J.D.A. designed and directed the study. Competing interests: J.H.V., L.H., N.E., and J.D.A. are inventors on patent applications filed by the University of Southern Mississippi on the polymer and devices described in this work. Data and materials availability: All data needed to evaluate the conclusions in the paper are present in the paper and/or the Supplementary Materials.

Submitted 19 December 2020

Accepted 21 April 2021

Published 9 June 2021

$10.1126 /$ sciadv.abg2418

Citation: J. H. Vella, L. Huang, N. Eedugurala, K. S. Mayer, T. N. Ng, J. D. Azoulay, Broadband infrared photodetection using a narrow bandgap conjugated polymer. Sci. Adv. 7, eabg2418 (2021). 


\section{ScienceAdvances}

\section{Broadband infrared photodetection using a narrow bandgap conjugated polymer}

Jarrett $\mathrm{H}$. Vella, Lifeng Huang, Naresh Eedugurala, Kevin S. Mayer, Tse $\mathrm{Nga} \mathrm{Ng}$ and Jason D. Azoulay

Sci Adv 7 (24), eabg2418.

DOI: $10.1126 /$ sciadv.abg2418

ARTICLE TOOLS

SUPPLEMENTARY

MATERIALS

REFERENCES

PERMISSIONS http://advances.sciencemag.org/content/7/24/eabg2418

http://advances.sciencemag.org/content/suppl/2021/06/07/7.24.eabg2418.DC1

This article cites 41 articles, 4 of which you can access for free http://advances.sciencemag.org/content/7/24/eabg2418\#BIBL

http://www.sciencemag.org/help/reprints-and-permissions

Science Advances (ISSN 2375-2548) is published by the American Association for the Advancement of Science, 1200 New York Avenue NW, Washington, DC 20005. The title Science Advances is a registered trademark of AAAS.

Copyright @ 2021 The Authors, some rights reserved; exclusive licensee American Association for the Advancement of Science. No claim to original U.S. Government Works. Distributed under a Creative Commons Attribution License 4.0 (CC $\mathrm{BY})$. 